Tatiana A. Sénina (moniale Kassia)

Saint-Petersburg, Russia mon.kassia@gmail.com

\title{
LA BYZANCE TIRÉE DE LA CLANDESTINITÉ
}

\begin{abstract}
Сергей А. ИвАнов, В поисках Константинополя. Путеводитель по византийскому Стамбулу и окрестностям (Москва: Вокруг света, 2011) 752 с., ч/б идлюстрации, карты, схемы. ISBN 978-5-98652-382-8
\end{abstract}

Serge A. Ivanov, En quête de Constantinople. Guide de l'Istanbul byzantin et de ses environs (Moscou : Vokrug sveta, 2011) 752 p., illustrations en noir et blanc, cartes, schémas.

C'est un livre que beaucoup d'admirateurs de l' « Eil de l'univers » brûlaient de voir, et voici la réalisation de ce désir. Réalisation vraiment magnifique! On lit ce guide comme un roman passionnant et, après avoir tourné la dernière de ses 752 pages, on regrette qu'elles aient touché trop vite à leur fin.

Devant nous le guide uniquement de la Constantinople byzantine : aucune information sur les hôtels, restaurants, magasins etc., rien sur les monuments turcs et même sur les églises grecques contemporaines, - tout cela n'est mentionné que dans le cas où quelques ruines byzantines se trouvent en-dessous ou s'y trouvaient autrefois. C'est un guide pour ceux qui s'intéressent au passé byzantin d'Istanbul et qui veulent savoir tout et voir tout ce qui y reste de la Byzance.

L'œuvre de S. A. Ivanov répond parfaitement à ce but : des cartes détaillées et des indications claires permettront d'atteindre tous les monuments byzantins qui restent dans la Ville et ses environs (au Bosphore, sur toutes les côtes et les îles de la Propontide, dans la Thrace jusqu'à Visé), et les schémas des monuments les plus importants (tels que Sainte Sophie ou Saint-Sauveur-in-Chora) aideront à voir tout ce que l'on peut trouver des restes byzantins, y compris beaucoup de choses qui ne sont pas habituellement indiquées ni mentionnées dans les guides standard. L'auteur a visité lui-même, et souvent bien de fois, presque tous les endroits évoqués dans son guide, il a étudié une grande quantité de matériaux sur Constantinople, ses monuments et la vie de ses habitants, et «En quête de Constantinople » représente le résultat d'un travail soigneux de 10 ans - un résultat fort impressionnant. J'ai visité la Ville moi-même plusieurs fois déjà et j'étais sûre que j'avais 
vu presque tout ce qu'il y avait à voir, mais après avoir lu ce guide j'ai réalisé qu'il y reste encore une grande quantité de choses!

"J'aime les histoires "savoureuses" », dit l'auteur, « et je vous les raconterai telles qu'elles sont parvenues dans les sources» (p. 7). Ces nombreux faits historiques, croyances populaires, légendes et ragots tirés des chroniques, vies de saints, lettres, homélies, épigrammes etc, racontés ou cités en rapport avec tel ou tel monument, telle ou telle place historique rendent ce guide très vif et extraordinairement intéressant. Par exemple, dans la description du Forum de Constantin nous trouvons l'histoire de la mort du célèbre hérétique Arius qui a expiré dans les toilettes publiques de ce même forum (pp. 256-257); dans le récit sur l'église de Myrélaion on peut apprendre comment $\mathrm{S}$. André a démasqué un voleur (pp. 273-275) ; en rapport avec les inhumations dans le monastère de Chora (Kariye Camii) l'auteur nous cite quelques épitaphes et épigrammes écrits par «Manuel Philes, le poétereau principal de cette époque » (pp. 505-506, 509-510). Serge Ivanov connait parfaitement le matériel, et la Ville de Constantin avec ses habitants, dépeinte par sa plume, se présente devant nous non comme morte depuis longtemps et mal compréhensible, mais comme tout à fait vivante et proche de l'homme contemporain dans beaucoup d'aspects. En grimpant les ruines byzantines, plus ou moins mal conservées, en admirant les mosaïques et les églises ou leurs restes, «tu comprends que la Byzance n'est pas disparue - elle est passé dans la clandestinité » (p. 10).

Le guide comporte une Préface (pp. 5-16) et seize chapitres : «En face des aveugles » - une courte histoire de la Ville (pp. 17-25); « Sainte Sophie » (pp. 26-84) ; «Le Grand palais » (pp. 85-138); «L'Acropole » (pp. 139-181); «Le Musée Archéologique » (pp. 182-210); «Le sud-est » - le Palais d'Antioche, S. Euthemie, l'Hippodrome, SS. Serge et Bacchus, la Mésé, le Forum de Constantin, les Artopoleia, le Myrélaion etc (pp. 211-280) ; « Le nord-est » - la citerne Basilique, les Chalcoprateia, le Néorion, les factoreries italiennes, Kilise Camii, Kalenderhane, S. Polieucte et d'autres (pp. 281-326) ; "Le nord-ouest» - le monastère de Lips, la quatrième colline avec les SS. Apôtres, l'aqueduc de Valens, le Pantocrator, Eski Imaret Camii, S. Théodosie, les murs le long de la Corne d'Or, le Phanar et quelques églises (pp. 327-379) ; « Le sud-ouest » - le Stoudios, les quartiers Psamthion, Gastria et d'autres, le Forum d'Arcadius (pp. 380-418); «Les Blachernes » (pp. 419-470); «Kariye Camii » (pp. 471-511) ; «Les murs de Théodose» (pp. 512555); «Le Bosphore, Galata et les Îles de Princes» (pp. 556-597); 
«L'ouest » - les aqueducs de Thrace, les murs d'Anastase, Vizé, Heracleia de Thrace, Sylimbrie, Hébdomon etc (pp. 598-639) ; « L'est » Chalcédoine, Nicomédie, Bryas et les autres banlieues asiatiques (pp. 640-677) ; "Le sud» - S. Abercius, SS. Archanges, le GrandChamp, le Médikion et d'autres grands monastères, Nicée, Panormos, Cyzique etc (pp. 678). Chaque partie est munie d'une bibliographie avec la littérature scientifique.

Les nombreux déchiffrements des inscriptions byzantines conservées sur les murs, mosaïques, obélisques etc., contenus dans cette édition, représentent un intérêt particulier.

On trouvera dans le guide beaucoup d'illustrations en noir et blanc: photographies de monuments, de mosaïques et de monnaies, reproductions de miniatures byzantines.

Après la Conclusion (pp. 729-733) le lecteur trouvera quelques informations utiles : les horaires d'ouverture des Musées, un petit glossaire de termes spéciaux, la liste des empereurs byzantins, les tables généalogiques et un index des noms propres.

À regret, le livre n'est pas privé de quelques défauts, d'ailleurs presque inévitables dans les œuvres aussi grandes. Certains de ces défauts sont liés à l'utilisation non critique des traductions russes des sources byzantines. Ainsi, une erreur fâcheuse se trouve sur la première page de la Préface même du guide : en racontant l'histoire du meurtre de l'empereur Léon V l'Arménien, Ivanov cite (p. 5) la traduction russe du Continuateur de Théophane fait par Ya. N. Lubarskiy où la première ligne de l'hirmos du $2^{\text {ème }}$ canon de la Nativité du Christ est traduite

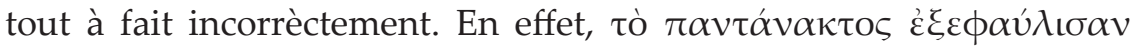
тó $\Theta \omega$ du Continuateur ${ }^{1}$ est traduit par Lubarskiy comme « Отрешили страстью Всевышнего » (« [Ils] ont révoqué par la passion du Très Haut»), ce qui est absolument absurde. Le Continuateur cite bien sûr la première ligne de l'hirmos de la $7^{\text {ème }}$ ode du canon ïambique écrit par S. Jean Damascène :

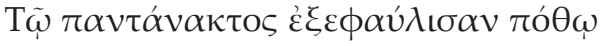

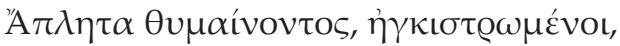

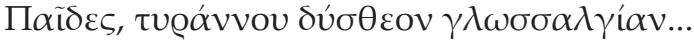

C'est-à-dire (je souligne les mots cités chez le Continuateur) :

(1) Theophanes Continuatus, Chronographia, dans: I. BEKKER (ed.), Theophanes Continuatus, Ioannes Cameniata, Symeon Magister, Georgius Monachus (Bonn, 1838) (Corpus Fontium Historiae Byzantinae) 39.2. 
«Saisis d'amour pour le Roi de tout, les adolescents ont méprisé le bavardage impie du tyran terriblement furieux... $»^{2}$

La traduction slavonne de ce même texte :

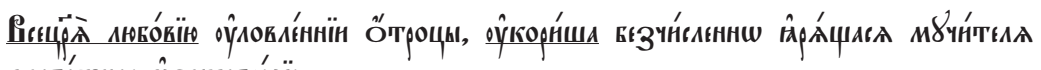

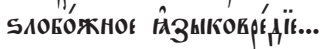

Ainsi, dans la traduction russe des premières lignes de cet hirmos il faudrait citer «Всецаря любовью уловленные ( «Saisis d'amour pour le Roi de tout », ce qui correspond à la première ligne de la traduction slavonne) ou bien, plus exactement, «За Всецаря любовь презрели » («Pour l'amour du Roi de tout [ils] ont méprisé »). Bien sûr dans les traductions slavonnes de l'hymnographie byzantine il y a un certain nombre d'erreurs et de passages obscurs, mais ce n'est pas le cas des originaux, et c'est assez étrange qu'une traduction aussi absurde n'ait engendré jusqu'à présent aucune question. Il est tout à fait regrettable que cette erreur faite dans la première édition de la traduction russe se soit déplacée dans la deuxième, et que nous la voyions maintenant dans cet admirable guide.

Dans le guide il y a également quelques inexactitudes historiques. Par exemple, Serge Ivanov écrit qu'en 816-817, S. Théodore le Stoudite était emprisonné dans le monastère Saints Serge et Bacchus (p. 245), mais ce n'est pas correct: le leader des iconophiles a passé ce temps en exil assez loin de Constantinople, ${ }^{3}$ et c'est son élève Naucrace le Stoudite qui était prisonnier dans ce monastère. ${ }^{4}$ L'année 829 comme date du choix de fiancée pour l'empereur Théophile, dans lequel la poétesse Cassia a participé (p. 327), semble un peu étrange. L'avènement de Théophile au trône a eu lieu en octobre 829, et si le choix de

(2) La traduction française de ce texte faite par le P. Denis Guillaume ( Epris d'amour divin pour le Roi de l'univers, les Jeunes Gens ont méprisé le bavardage impie d'un tyran à l'insatiable orgueil ») et parue en 1980 (dans le Ménée du décembre, édition de la «Diaconie apostolique ») je trouve inexacte.

(3) Voir Th. Pratsch, Theodoros Studites (759-826) - zwischen Dogma und Pragma. Der Abt des Studiosklosters in Konstantinopel im Spannungsfeld von Patriarch, Kaiser und eigenem Anspruch (Frankfurt am Main-Berlin-Bern-New York-Paris-Wien, 1998) (Berliner byzantinistische Studien, 4) 247-256.

(4) Théodore l'écrit dans une lettre à Naucrace, d'où nous connaîssons que Naucrace était emprisonné et avait eu une discussion avec Jean le Grammairien sur les icônes, voir G. Fatouros (ed.), Theodori Studitae Epistulae (Berlin-New York, 1992) (Corpus Fontium Historiae Byzantinae, Series Berolinensis, 31) Ep. 380 (la fin de 817 ou le début de 818). 
fiancée se fut passé vraiment après la mort de Michel II et non pas en 821 quand Théophile est devenu co-empereur, on aurait du indiquer la date de $830 .^{5}$

On peut aussi noter quelques défauts dans la mise en pages du livre. Par exemple, sur les pages 86-87 les numéros des monuments sont indiqués dans la liste mais manquent sur la carte. Sur les pages 216-220 les titres de colonne sont confondus : «Евфимия » au lieu de « Ипподром. Карцеры ». Mais dans son ensemble, la mise en pages est de bonne qualité.

À mon avis, le plus grand défaut de ce guide est sa table des matières : elle ne contient que les titres des chapitres, mais les titres des subdivisions manquent, ce qui rend assez difficile l'orientation dans le livre. Je pense aussi qu'il faudrait munir le guide d'un index des monuments qui y sont mentionnés.

Peut-être certains lecteurs trouveront quelque peu inconvenable le ton de l'auteur qui semble parfois trop familier par rapport aux byzantins, y compris les empreurs et les patriarches. Néanmoins, je trouve que ce procédé est justifié : c'est un moyen d'approcher ces gens, qui semblent être si éloignés de nous, et de montrer qu'en réalité les byzantins ressemblent à nos contemporains plus que nous ne le pensions.

Je suis sûre que les admirateurs de la Byzance remercieront maintes et maintes fois Serge Ivanov pour son œuvre merveilleuse.

(5) Cette date est soutenue par W. T. Treadgold (The Bride-Shows of the Byzantine Emperors, Byzantion 49 (1979) 395-413; IDEM, The Historicity of Imperial Bride-Shows, Jahrbuch der Österreichischen Byzantinistik 54 (2004) 39-52), mais, vérification faite, ses arguments sont peu concluants, et la date de 821 semble plus probable (voir R.-J. Lilie, C. Ludwig, Th. Pratsch, I. Rochow (Hrsg.), Prosopographie der Mittelbyzantinischen Zeit. Erste Abteilung (641-867) (Berlin, 1998) \# 7286, p. 344 et 347 n. 1, \# 8167, p. 629 et 632 n. 1 ; Т. А. Сенина (монахиня Кассия), Диалог Феофила и Кассии: дитературная выдумка или реальность?, Scr 2 (2006) 240-272, surtout 249-263). 